\title{
Laboreal
}

Volume $2 \mathrm{~N}^{\circ} 1$ | 2006

Varia

\section{Percepción del riesgo de exposición al ruido}

Percepção do risco de exposição ocupacional ao ruído

Perception du risque d'exposition au bruit en milieu professionnel

Perception of the risk of occupational noise exposure

\section{Pedro Arezes}

\section{OpenEdition}

\section{Journals}

Edición electrónica

URL: http://journals.openedition.org/laboreal/13754

DOI: 10.4000/laboreal.13754

ISSN: 1646-5237

\section{Editor}

Universidade do Porto

Referencia electrónica

Pedro Arezes, «Percepción del riesgo de exposición al ruido », Laboreal [En línea], Volume 2 №1 | 2006, Publicado el 01 julio 2006, consultado el 24 septiembre 2020. URL : http://journals.openedition.org/ laboreal/13754; DOI : https://doi.org/10.4000/laboreal.13754

Este documento fue generado automáticamente el 24 septiembre 2020

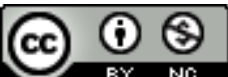

Laboreal está licenciado com uma Licença Creative Commons - Atribuição-NãoComercial 4.0 Internacional. 


\title{
Percepción del riesgo de exposición al ruido
}

\author{
Percepção do risco de exposição ocupacional ao ruído \\ Perception du risque d'exposition au bruit en milieu professionnel \\ Perception of the risk of occupational noise exposure
}

\section{Pedro Arezes}

\section{REFERENCIA}

Arezes, P. (2002). Percepção do risco de exposição ocupacional ao ruído. Tese de Doutoramento, Escola de Engenharia da Universidade do Minho, Guimarães.

\section{NOTA DEL EDITOR}

Manuscrito recibido en : febrero/2006

Aceptado tras peritage en : mayo/2006

\section{Introducción}

1 El estudio de la relación entre el trabajo y la salud implica una correcta identificación de los factores ocupacionales en juego, así como de sus repercusiones, positivas o negativas, sobre los trabajadores. Para eso, es indispensable la realización de estudios prácticos incidiendo sobre los contextos reales de trabajo, identificando en estos las principales condicionantes del riesgo ocupacional.

2 Entre los varios factores de riesgo ocupacional tiene particular importancia la exposición a niveles de presión sonora elevados, dada su frecuencia en ambientes industriales. La exposición ocupacional al ruido ha sido bastante estudiada a lo largo de 
los años constituyendo, no obstante, una de las principales causas de enfermedad profesional, la hipoacusia neuro-sensorial derivada del ruido, la cual abarca, según datos oficiales, alrededor de $25 \%$ de los trabajadores con incapacidad.

Por la observación de las prácticas reales de trabajo, se comprueba que los trabajadores, aunque desempeñando idénticas funciones en los mismos puestos de trabajo, tienen concepciones diferentes de los riesgos a los que están expuestos. En el caso de la exposición ocupacional al ruido, esas discrepancias son aún más evidentes. Así, es frecuente encontrar trabajadores compartiendo el mismo puesto de trabajo, divergiendo, sin embargo, sobre la forma como ven el riesgo de exposición al ruido, o la forma como piensan que este les afecta.

4 En lo que se refiere a la exposición al ruido, existen varios abordajes de comprensión de la relación entre la percepción del riesgo y el uso de la Protección Individual Auditiva (PIA). Sin embargo, una gran parte de estos abordajes carecen de análisis cuantitativos de factores centrales, tales como, los niveles de presión sonora a los que los trabajadores están expuestos y las pérdidas auditivas que estos presentan.

\section{Riesgo y Percepción}

5 En el dominio ocupacional, el riesgo es habitualmente considerado como siendo una función de dos factores principales, la probabilidad de un evento y la gravedad potencial a él asociada. Así, la cuantificación del riesgo asociado a determinado evento, por ejemplo la ocurrencia de un accidente, será función de la probabilidad de que este ocurra y de la gravedad de que esta ocurrencia pueda acarrear.

6 La cuantificación objetiva del riesgo, en términos ocupacionales, representa una tarea compleja dado el conjunto de condicionantes asociados a la probabilidad y a la gravedad del riesgo considerado. Sin embargo, para algunos factores de riesgo ocupacional, como es el caso del ruido, es posible establecer valores-limite para un determinado parámetro físico cuya cuantificación sea posible.

7 Pero si, por un lado, tenemos la cuantificación objetiva del riesgo, midiéndolo a través de equipos de medida más o menos precisos, por otro tenemos la forma como los trabajadores miran hacia ese riesgo. Y esta forma de ver el riesgo, o su percepción, podrán llevar a situaciones tan variadas cuanto la sobrestimación del riesgo, incluso cuando él es residual, o su subestimación, incluso cuando él está manifiestamente presente.

8 La percepción del riesgo es un término usado para referir el conocimiento y el sentimiento asociados, incluyendo las potenciales consecuencias, a una situación o a un conjunto de circunstancias. El estudio de la percepción del riesgo tuvo su inicio a finales de los años 1960, con la formulación por Starr de la cuestión "How safe is safe enough ?" (Weyman \& Kelly, 1999). Desde entonces ha habido algunos desarrollos en términos cuantitativos, aunque la gran aportación para esta área esté sobretodo relacionado con el riesgo ambiental. Los estudios en contexto ocupacional han enfocado, con más frecuencia, la forma como las personas se comportan ante el riesgo y no la percepción del riesgo propiamente dicho. 


\section{Percepción del riesgo y exposición al ruido}

9 El esfuerzo concentrado en la mejora de las condiciones de trabajo tiene por objetivo reducir, o hacer desaparecer, los riesgos "objetivos". Sin embargo, la percepción del riesgo, así como, la evaluación subjetiva de las condiciones de trabajo y del ambiente ocupacional, podrán, según algunos autores (Nelson, Aylor \& Nelson, 1999), ser igualmente importantes para el comportamiento de los trabajadores en lo que toca al riesgo y, así, podrán influenciar el riesgo "objetivo" y la seguridad.

La percepción individual del riesgo parece ser un antecedente crítico del comportamiento de riesgo (Glendon \& McKenna, 1995 ; Diaz \& Resnick, 2000). La forma como los trabajadores perciben el riesgo al que están expuestos durante su trabajo podrá representar una aportación para una mejor comprensión de su gestión y, de esa forma, para la mejora de sus condiciones de trabajo (Rundmo, 1996).

11 Dados referidos en la literatura (Weyman \& Kelly, 1999) sugieren que la noción de "control", "familiaridad", "desfase del efecto" (retraso entre la exposición y el aparecimiento de los síntomas) y "características físicas observables" (por ejemplo el olor, el color, etc.) tienen igual probabilidad de desempeñar un papel importante en la percepción del riesgo por parte de los trabajadores expuestos, particularmente a substancias banales en el día-a-día de trabajo. La exposición al ruido tiene muchas de estas características, designadamente, la del desfase del efecto que podrá, de alguna forma, influenciar la percepción del individuo al riesgo específico.

Pese a que la prioridad que, consensualmente, se atribuye a la adopción de medidas de protección colectiva, de naturaleza técnica y organizativa, se comprobará que, en el ámbito de la lucha contra la exposición al ruido, las empresas atribuyen un papel de relieve a la adopción de medidas de protección individual. Sin embargo, los aspectos interrogados se refieren, en su mayoría con las características de atenuación de los protectores y con su puesta a disposición.

Por otro lado, y en lo que se refiere a la motivación para el uso de la protección individual, varios autores (Rabinowitz, Melamed, Feiner \& Weisberg, 1996 ; Lusk, Ronis \& Kerr, 1995) refieren que esta debe centrarse en determinadas variables, como por ejemplo, las variables personales, que abarcan la percepción de eficacia, la percepción de la susceptibilidad y la incomodidad provocada por el ruido.

Las normas de uso de la protección auditiva recaen a veces en una escala propia de evaluación del ruido, normalmente basada en el intercambio de opiniones con otros colegas y en su propia concepción de audición normal, en detrimento de mediciones objetivas del ruido e informaciones sobre el fenómeno de las pérdidas auditivas reveladas por los audiogramas.

15 El actual trabajo - habiendo incidido sobre una muestra de 516 trabajadores de varias empresas industriales, expuestos a niveles de presión sonora superiores al nivel de acción preconizado en la legislación nacional $(85 \mathrm{~dB}(\mathrm{~A}))$ - quiso analizar la relación entre la percepción individual del riesgo y el uso de protección PIA, así como, entre la primera y el desarrollo de pérdidas auditivas que transcurren de la exposición ocupacional al ruido. Para el efecto, se desarrollaron dos modelos conceptuales, con base en la revisión bibliográfica efectuada.

Considerando la revisión bibliográfica, se desarrolló un cuestionario para evaluación de las variables de naturaleza esencialmente cualitativa, designadamente, la percepción 
individual del riesgo, la percepción de los efectos del ruido, la expectativa/valoración de los resultados, la cultura de seguridad y el comportamiento de riesgo. Simultáneamente se efectuó un cuestionario con el objetivo de caracterizar el tipo de exposición ocupacional al ruido, así como, el uso de la protección individual auditiva.

Para cuantificación de la variable referente a las pérdidas auditivas se han utilizado los resultados de los ensayos audiométricos realizados a los trabajadores al inicio del turno de trabajo.

\section{Conclusiones}

La exposición al ruido constituye un factor de riesgo que, dada su naturaleza, es frecuentemente percibido como no siendo posible controlar, asociándose a esa exposición una resignación fatalista, con potenciales consecuencias al nivel del comportamiento.

Será seguramente complejo intentar entender, con claridad, el comportamiento de riesgo, designadamente la decisión de no usar PIA, sin un análisis a priori de los puestos de trabajo, pero también sin analizar las opiniones y actitudes de los trabajadores. El conocimiento de la percepción individual de los trabajadores sobre el fenómeno de exposición al ruido parece constituir un elemento clave para la definición de cualquier estrategia que vise la protección de los trabajadores.

Un aspecto a evidenciar de los resultados obtenidos se refiere al reducido número de usuarios de PIA, es decir, apenas 284 trabajadores (aproximadamente $55 \%$ del total) afirmaron utilizar PIA. Si aislamos los trabajadores que dicen utilizar la PIA todo el tiempo $(100 \%)$ este número es aún más reducido $(\mathrm{N}=137)$. Por otras palabras, apenas aproximadamente $27 \%$ de los trabajadores inquiridos refirieron utilizar la PIA todo el tiempo, aunque, según la legislación aplicable, esta debería se utilizada por la totalidad de los trabajadores inquiridos.

21 Este reducido uso de PIA es consistente con resultados encontrados en estudios similares (McCullagh, 1999; Brady, 1999). Aunque el objetivo principal de este estudio no dijera respeto a la evaluación de la eficiencia de los PIA, tal no podrá dejar de ser referido cuando se comprueba que, incluso cuando aislamos a los trabajadores que afirmaron usar protección auditiva, el porcentaje del tiempo de uso es insuficiente, siendo, en media, del $82,1 \%$ del tiempo de turno.

De hecho, se no interrogamos sobre el uso de la PIA en términos medios, y considerando la forma de cálculo de la atenuación dada por los protectores en función del tiempo de uso, comprobamos que incluso para protectores que presentan elevados valores de atenuación catalogada, por ejemplo de $40 \mathrm{~dB}$, la atenuación real no supera los 7,5 dB. Si, por otro lado, consideramos los valores medios de uso de la PIA en el total de la muestra ( $\mathrm{N}=516)$, el porcentaje medio pasa a ser de $45,2 \%$. En este último caso, se obtienen, para el ejemplo dado anteriormente, una atenuación de apenas 2,6 dB, es decir, la eficiencia de la PIA, en términos medios, es prácticamente nula.

El análisis estadístico de los resultados obtenidos en este trabajo, a través del uso de técnicas estadísticas de análisis multivariante (path analysis), sugiere que la percepción individual del riesgo, y otros factores con ella relacionada, constituye un aspecto relevante en el análisis del comportamiento de los trabajadores. 
En el análisis de los principales factores que condicionan el uso de la PIA, se destaca el efecto de mediación de las variables relativas a la percepción individual, en particular el efecto de la percepción individual del riesgo. El análisis del modelo conceptual, a través del análisis de regresión lineal múltipla, sugiere aún que la promoción del uso de la PIA, en el ámbito de un Programa de Conservación de la Audición, debe asentar, por un lado, en la actuación sobre la percepción individual de los trabajadores y, por otro lado, en la retirada de las "barreras" de disuasión o inhibidoras del uso de la PIA.

Por este motivo, la percepción del riesgo debe tenerse en cuenta en el planeamiento, desarrollo e implementación de los Programas de Conservación de la Audición, en especial en lo que se refiere al desarrollo de planes formativos.

El conjunto de resultados obtenidos por el análisis de los modelos considerados en este trabajo permite concluir que el reconocimiento, por parte de los trabajadores, del riesgo asociado a la exposición ocupacional al ruido, constituye un importante paso hacia una mejor prestación de Seguridad de las empresas de las cuales forman parte y, consecuentemente, para la mejora continua de sus condiciones de trabajo.

\section{BIBLIOGRAFÍA}

Brady, J. (1999). Training to promote worker's use of hearing protection : The influence of work climate factors on training effectiveness. PhD Thesis. Michigan State University, East Lansing.

Diaz, Y. \& Resnick, M. (2000). A model to predict employee compliance with employee corporate's safety regulations factoring risk perception. In Proceedings of the IEA2000/HFES2000 Congress, vol. 4, (pp. 323-326). IEA, San Diego.

Glendon, I. \& McKenna, E. (1995). Human Safety and Risk Management. London : Chapman \& Hall. Lusk, S., Ronis, D. L.,\& Kerr, M. J. (1995). Predictors of Hearing Protection Use Among Workers : Implications for Training Programs, Human Factors, 37, 3, 635-640.

McCullagh, M. (1999). Factors affecting hearing protector use among farmers. PhD. Thesis. University of Michigan, East Lansing.

Nelson, D., Aylor, B. \& Nelson, R. (1999). Development of a questionnaire to examine worker risk perception of noise and use of hearing protection devices. In AIHce Conference Abstracts, paper 19. Toronto, Canada.

Rabinowitz, S., Melamed, S., Feiner, \& M., Weisberg, E. (1996). Hostility and hearing protection behavior : the mediating role of personal beliefs and low frustration tolerance, Journal of Occupational Health Psychology, 1, 4, 375-381.

Rundmo, T. (1996). Associations between risk perception and safety, Safety Science, 24, 3 , 197-209.

Weyman, A. \& Kelly, C. (1999). Risk perception and risk communication : a review of the literature, Health and Safety Executive Contract Research Report No. 248/1999, United Kingdom : Health and Safety Executive (HSE). 
AUTOR

PEDRO AREZES

Universidade do Minho - DPS, Escola de Engenharia da Universidade do Minho 4800 - 058

Guimarães

parezes@dps.uminho.pt 